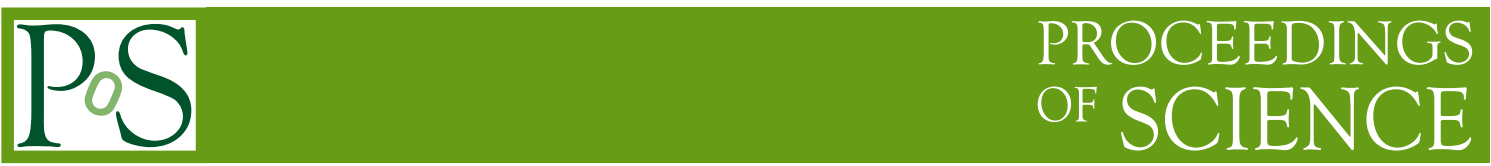

\title{
Investigations on MultiView VLBI for SKA
}

\author{
Richard Dodson* \\ ICRAR/UWA, Australia \\ E-mail: richard.dodsoneicrar.org \\ María Rioja \\ ICRAR/UWA (Australia), CASS/CSIRO (Australia), OAN/IGN (Spain) \\ E-mail: maria.rioja@icrar.org
}

The SKA will deliver orders of magnitude increases in sensitivity, but most astrometric VLBI observations are limited by systematic errors. In these cases improved sensitivity offers no benefit. The best current solution for improving the accuracy of the VLBI calibration is MultiView VLBI, where multiple simultaneous observations around the target are used to deduce the corrections required for the line of sight to the target. We have estimated and quantified the applicability of MultiView from real-world ionospheric studies, making projections into achievable astrometric accuracies. These predict systematic measurement errors, with calibrators separated by several degrees, of $\sim 10 \mu$ as with current VLBI facilities. For closer calibrators, that are in-beam for single dish VLBI facilities, we predict systematic measurement errors of a few $\mu$ as. This is the ideal combination, where the sensitivity of the SKA will provide the precision and MultiView will provide the accuracy. Based on these results we suggest that the SKA design should increase the number of VLBI beams it can form from four to as many as ten.

14th European VLBI Network Symposium \& Users Meeting (EVN 2018)

8-11 October 2018

Granada, Spain

${ }^{*}$ Speaker. 


\section{Introduction}

Very Long Baseline Interferometry (VLBI) can result in the highest angular resolutions achievable in astronomy and has a unique access to emission regions that are inaccessible with any other approach. One of the most powerful applications of VLBI is in the measurement of high precision relative astrometric angles between sources. Because the reference sources are (usually) Quasars (QSOs) at high red shifts measurement of parallaxes with accuracies of $\sim 10 \mu$ asare possible, sufficient for measurement of distances across the span of our Galaxy. Phase referencing between sources allows for the precise correction of the atmospheric contributions, which are typically $3 \mathrm{~cm}$ of wet delay from the troposphere and 6TECU of free-electron plasma from the ionosphere [see, for example, 1].

Nevertheless the applications of astrometric-VLBI are not widespread outside of the centimeter wavelengths, say $5-22 \mathrm{GHz}$. The observations become progressively more challenging away from these frequencies. For the lower frequencies the chief challenge is that the ionospheric distortions are wildly different for different lines of sight.

The SKA collecting area, and thus the sensitivity, will be two orders of magnitude larger than current facilities. Therefore when cross correlated with existing VLBI facilities there will be an order of magnitude increase in VLBI sensitivity. Furthermore when combined with the largest current VLBI facilities, such as $100 \mathrm{~m}$ or above (e.g. the 500m FAST dish in China), it will be possible to achieve very close to the two orders of magnitude sensitivity gains. The astrometry achievable with the thermal sensitivity limits from such an interferometer are of the order of 10 to $1 \mu$ as (i.e. a dynamic range of 1,000 to 10,000 ), depending on the size, number and configuration of the antennas. The challenge is to reduce the systematic errors to the same order, and this requires the development of innovative astrometric techniques that will address the underlying limitations for the SKA frequencies.

\section{New Astrometric Techniques}

Current best-practise astrometric performance is currently at $22 \mathrm{GHz}$ (e.g. the results achieved in the BeSSeL project [2]), where the troposphere dominates the astrometric budget. BeSSeL developed and makes extensive use of geodetic blocks to reduce the astrometric error from the static troposphere by a factor of three. A $1 \mathrm{~cm}$ error on a $6000 \mathrm{~km}$ baseline provides relative astrometric accuracy of $\sim 10 \mu$ as, with the calibrator at $1^{\circ}$ separation.

The best $1.6 \mathrm{GHz}$ results come from finding very nearby calibrators; ionospheric residuals are typically 6TECU, which the equivalent of a position error of $\sim 100 \mathrm{~cm}$, at these frequencies. Close calibrators dilute the errors linearly as a function of separation (if the results are not dominated by thermal errors). Therefore, for $10 \mu$ as accuracy in the presence of $100 \mathrm{~cm}$ errors, one would require a separation of about $1^{\prime}$. The PSRPi project [3] has demonstrated great success by finding calibration sources extremely close to the targets. These tend to be without well-known VLBI positions and weak (requiring a longer calibration solution interval). The median separation was $14^{\prime}$, with one out of 70 at $1^{\prime}$. The median per-epoch accuracy of these observations was about $100 \mu$ as. Note that the median final parallax accuracy (based on about 9 observations) was $60 \mu$ as, even for the closest pairing. In this case the weak calibrator source meant that the increased thermal noise dominated 
the very small systematic error. The PSRPi analysis was consistent with a quadratic combination of thermal errors plus a linear dependence on the separation, as in Asaki etal. [1].

The only SKA requirements for conventional in-beam calibration are i) great sensitivity to allow for more (and therefore closer) calibrators for any arbitrary line of sight and ii) the ability to form two tied-array beams if the SKA tied-array beam size is smaller than the source separation, as is highly likely. But PSRPi found only one calibrator as close as $1^{\prime}$ from the 70 PSR targets. As a SKA-VLBI baseline is the order of 10 times more sensitive we could only expect a source as close as $1^{\prime}$ in $\sim 10 \%$ of cases. This is inline with the analyses in Godfrey and Imai etal. ([5], [6]).

MultiView takes a new approach, by measuring the 2D phase surface over the antenna site [10]. One measures the calibration terms in the direction of a number of calibrators around the target and projects those values to the actual line of sight to the target. These sources can then be both stronger and further from the target. The former allows for shorter solution intervals and smaller thermal errors. For a planar surface with perfect calibrators one would require 3 measurements to solve for the target direction. This was the basis of our previous recommendation for a minimum of 4 VLBI beams for SKA. We can think of no example where there is a benefit from taking calibrators outside the primary beam of the SKA antennas $(\sim 1 \mathrm{deg})$ for SKA-VLBI. We focus on the case were the conventional VLBI antennas have the sources in-beam (i.e. a fraction of a degree for most cases) and the SKA ties all its stations together with multiple tied-array beams. We label this mode as 'in-beam MultiView' as opposed to MultiView with conventional VLBI switching.

\section{Crucial questions for SKA}

The crucial design considerations for VLBI-SKA, with respect to MultiView astrometry are:

- How many beams are needed to reduce the systematic errors to that of the thermal errors?

- Would more beams allow fitting a curved surface?

- Would more beams allow contemporaneous checks on calibrators?

Initially the requirements were to form four beams, which is the minimum to measure calibrators in three directions, and to form a linear combination to solve for the target direction. But how reliable is that assumption, and is it sufficient for achieving the astrometric goal of $10 \mu$ as in a single epoch? The latter comes from the desire to match the systematic errors to the thermal errors in an observation with a SNR ratio of 1000, which would easily be achievable with VLBI observations between an SKA Phase-1 core and current VLBI resources. Increasing the number of beams would allow for solving for non-linear ionospheric surfaces and for more careful cross checking of calibrators. Monitoring the latter to uncover unexpected changes in the reference position, which would be revealed by the shift of one particular calibrator against the others.

To answer these questions we turned to two datasets, both from the Murchison Widefield Array (MWA). The first is the output from the Real Time System (RTS), which can be used to provide calibration information on the array with solutions every 8 seconds [8]. The shift in the positions of known sources provides us with a measure of the average TEC gradient over the array. The downside of this is that the average gradient over the array does not provide the fine scale structure of the TEC surface. For a $3 \mathrm{~km}$ array (as for these data) and an ionospheric screen at a height of $300 \mathrm{~km}$ the minimum angular scale is $\sim 0.6^{\circ}$. The results from RTS are most relevant to phase referencing with current VLBI arrays. We have taken 58 examples of the RTS dataset representing the four atmospheric conditions identified by Jordan etal. [7]. This dataset contain 
one day each of: 'weak' ionospheric structure (type 1), moderately correlated (type 2), highly correlated but weak (type 3) and highly correlated and strong (type 4). Jordan etal. [7] provided an absolute characterisation of the ionospheric conditions. Our analysis was more focused on the relative differences, which is that required for relative phase referencing.

For finer scale information, we turn to the by-product of the calibration method LEAP [9]. This method provides the station-based calibration all visible calibrators, in parallel. LEAP offers an extremely promising solution for direction dependent (DD) calibration at low frequencies. It does not require a sky model, just calibrator positions, and therefore the DD calibrations are independent, allowing real-time and parallel solutions. The station-based solutions can be used to characterise the smoothness of the TEC screen at the sub-degree scale. This is the regime most relevant to (phase referencing with) the SKA (to in-beam sources for the single dish antennas). We have taken the calibration solutions from 18 Dec 2017, which has been classified as moderate weather. All of these datasets were from the MWA Phase- 2 array, with a maximum baseline of about $6 \mathrm{~km}$.

\subsection{RTS analysis}

MultiView phase referencing will work perfectly if the TEC gradients (no matter how large) are the same in the different calibrator directions, because the correction is linear. Therefore we are interested in the variance in the apparent shift in source positions as a function of angular separation, for all separations. We convert the measured shifts into the TEC gradient, which we express in TECU per degree. The mean value of the differences will capture curvature (the differential of the TEC gradients, $d \Delta \mathrm{TEC}$, over the FoV); for the variance we used the Root Mean Squared (RMS) value.

$$
\left.d \Delta \mathrm{TEC}_{i j} \propto \mathrm{P}_{i}-\mathrm{P}_{j} ; \sigma \Delta \operatorname{TEC}(\Omega)\right)=\sqrt{\sum_{i-j=\Omega} \overline{d \Delta \mathrm{TEC}_{i j}^{2}}}
$$

where $\mathrm{P}_{i}$ is the position offset of the $i^{\text {th }}$ source and $\Omega$ is the angular separation between the $i^{\text {th }}$ and $j^{\text {th }}$ source. The $1 \mathrm{D}$ behaviour of the variance provides an estimate as to how far we can typically trust a MultiView calibrator strategy for angular separations of one to tens of degrees.

\subsection{LEAP analysis}

LEAP analysis is fully described in Rioja etal. [9]; here we limit ourselves to the analysis of the measured fine-scale TEC surfaces from LEAP. The $\triangle$ TEC is measured for all calibrator directions across the field of view, after direction-independent and bandpass calibration. These individual TEC surfaces are a projection of the TEC onto the array and represent angular scales between the minimum and maximum baseline length $(10-6000 \mathrm{~m})$ over the height of the ionosphere. We can use concurrent measurements of the ionospheric screen height from, for example the space weather service (www.sws.gov.au), or - with little loss of applicability - one can assume a canonical $300 \mathrm{~km}$. This gives angular scales 0.1 to $60^{\prime}$. We assume that the measured TEC surface can be approximated by a planar surface (or a low-order polynomial) and measure the residuals. Assuming that the phase structure is a mixture of a simple structure plus turbulent noise we can discover i) how accurate a linear fit would be in predicting the residual TEC in an arbitrary target direction and ii) how often a higher order fit would be a significant improvement in that prediction. Because it is vital to obtain results with the smallest possible intrinsic noise we limited our selves to the strongest sources in each field of view. 


\section{Results}

For the large angular scales we find that the four classes do have noticeably different behaviour, as shown in Fig. 1. In type 1 the variance rose weakly with angular separation, with an average value of about $0.018 \mathrm{TECU} /{ }^{\circ}$, in type 2 the variance rose significantly with angular separation, from $\sim 0.018$ at small angular separations to nearly double that, at separations of $25^{\circ}$. Type 3 rose from from $\sim 0.018$ at small angular separations and plateaued at about $0.022 \mathrm{TECU} /{ }^{\circ}$ between 5 and $20^{\circ}$. Type 4 rose rapidly between 1 and $2^{\circ}$ to plateau at $0.05 \mathrm{TECU} /{ }^{\circ}$.

For the small angular scales we use the LEAP results to compare the residual RMS across the TEC (phase) surface against the expected value, given the source strength. Figure 2 shows this distrubtion for 1 st order (planar), 2nd order and 3rd order fitting. The distribution is clearly approximately Gaussian, with a mean close to one and $\sigma$ close to 0.14 . However $14 \%$ of the planar fit residuals have a fractional RMS is higher than $3 \sigma$. See Table 1 . These identify the TEC surfaces with significant curvature.
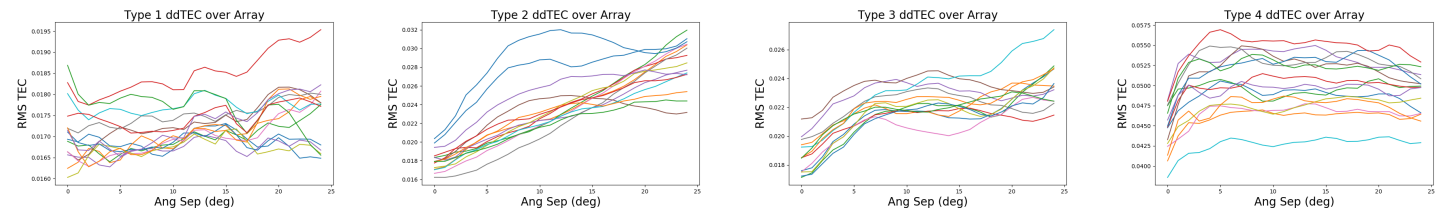

Figure 1: 1-D projection of the variance (normalised by the separation) as a function of angular separation. The four classes identified in Jordan etal. [7] are represented, running from type 1 to 4, left to right.

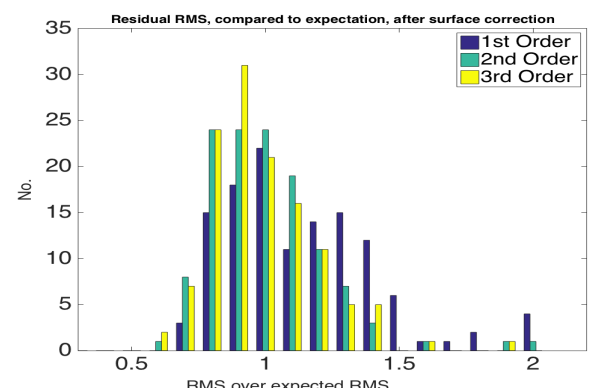

\section{Conclusions}

At large angular scales ionospheric types 1,2 and 3 would give acceptable astrometric results in MultiView VLBI with current facilities, even at low frequencies. A TEC error of 0.02TECU is equivalent to $0.4 \mathrm{~cm}$ at $1.4 \mathrm{GHz}$. But note that these residuals are the imperfections from a planar surface, so the 'dilution factor' of the angular separation do not apply. Therefore even the type 3 behaviour (highly correlated but weak) would allow astrometry at the $140 \mu$ as level, for a set of calibrators with an average offset from the target of a degree. Obviously if the separations are double that, the errors are also doubled. This is compatible with the results from [10], where we found errors of $\sim 100 \mu \mathrm{as}$. At higher frequencies, for example for $6.7 \mathrm{GHz}$ methanol masers (as being targeted by the BeSSeL project to extend the coverage of the galactic plane), we predict that in almost any conditions MultiView accuracies would exceed those of observations with the conventional geodetic blocks. 
Turning to the smaller angular scales, for the SKA in-beam MultiView VLBI case, we find: i) the source count estimates show the calibrator density will be insufficient to match the desired key-science goal of $10 \mu$ as, in $90 \%$ of cases. ii) That fitting planar surfaces give good solutions in $86 \%$ of cases, with residuals as low as 2 mTECU. At this level one matches the potential thermal limits, which is equivalent to the ionospheric delay being $\sim 1 \mathrm{~mm}$ (at $1.4 \mathrm{GHz}$ ). The remaining $14 \%$ have significantly higher residuals, which can be reduced to the same level by fitting higher order polynomials. iii) At the level of $1 \mu$ as source stability will become a significant limitation, as this has only been demonstrated to $\sim 10 \mu$ as level [e.g. 4]. The only generally practical method to address this is to have more than the minimum number of calibrators.

We conclude that: In-beam astrometry will not achieve the target levels of $10 \mu$ as per epoch in $\sim 90 \%$ of cases; Four beam MultiView-VLBI will be able to reduce the errors from planar ionospheric contributions and allow us to achieve the target levels; Four beam in-beam MultiViewVLBI with the SKA-mid could achieve systematic errors of $\sim 1 \mu$ as, in $\sim 90 \%$ of cases; Calibrator instability will be a major source of error at this level of accuracy, requiring extra calibrators and beams; Higher order fits to the TEC phase surface would allow systematic errors of $\sim 1 \mu$ as in nearly all cases.

Therefore, to achieve the theoretical astrometric accuracy of the SKA-VLBI of $\sim 1 \mu$ as, the number of beams of SKA-VLBI should be increased from the current four to at least six, and potentially ten. This will provide robustness against the ultimate limit of non-linear ionospheric surfaces and/or calibrator instability.

Acknowledgements: We thank C. Jordan for sharing the RTS datasets used in the analysis.

\section{References}

[1] Y. Asaki, et al., Verification of the Effectiveness of VSOP-2 Phase Referencing with a Newly Developed Simulation Tool, ARIS. PASJ, 59:397-418, April 2007.

[2] A. Brunthaler, et al., The Bar and Spiral Structure Legacy (BeSSeL) survey: Mapping the Milky Way with VLBI astrometry. Astronomische Nachrichten, 332:461, June 2011.

[3] A. T. Deller, et al., Microarcsecond VLBI pulsar astrometry with PSR $\pi$ II. parallax distances for 57 pulsars. arXiv e-prints, August 2018.

[4] E. Fomalont, K. Johnston, A. Fey, D. Boboltz, T. Oyama, and M. Honma. The Position/Structure Stability of Four ICRF2 Sources. AJ, 141:91, March 2011.

[5] L. Godfrey, H. Bignall, and S. Tingay. Very High Angular Resolution Science with the SKA. Technical report, Curtain University, Australia, May 2011.

[6] H. Imai, R. A. Burns, Y. Yamada, N. Goda, T. Yano, G. Orosz, K. Niinuma, and K. Bekki. Radio Astrometry towards the Nearby Universe with the SKA. arXiv e-prints, March 2016.

[7] C. H. Jordan, et al., Characterization of the ionosphere above the Murchison Radio Observatory using the Murchison Widefield Array. MNRAS, 471:3974-3987, November 2017.

[8] D. A. Mitchell, et al., Real-Time Calibration of the Murchison Widefield Array. IEEE Journal of Selected Topics in Signal Processing, 2:707-717, November 2008.

[9] M. J. Rioja, R. Dodson, and T. M. O. Franzen. LEAP: an innovative direction-dependent ionospheric calibration for low-frequency arrays. MNRAS, 478:2337-2349, August 2018.

[10] M. J. Rioja, R. Dodson, G. Orosz, H. Imai, and S. Frey. MultiView High Precision VLBI Astrometry at Low Frequencies. AJ, 153:105, March 2017. 